\title{
Riesz Transform Characterization of Weighted Hardy Spaces Associated with Schrödinger Operators
}

\author{
Hua Zhu \\ Beijing International Studies University, Beijing 100024, China \\ Correspondence should be addressed to Hua Zhu; zhuhua@pku.edu.cn
}

Received 1 March 2016; Accepted 8 June 2016

Academic Editor: Dashan Fan

Copyright ( 2016 Hua Zhu. This is an open access article distributed under the Creative Commons Attribution License, which permits unrestricted use, distribution, and reproduction in any medium, provided the original work is properly cited.

We characterize the weighted local Hardy spaces $h_{\rho}^{1}(\omega)$ related to the critical radius function $\rho$ and weights $\omega \in A_{1}^{\rho, \infty}\left(\mathbb{R}^{n}\right)$ by localized Riesz transforms $\widehat{R}_{j}$; in addition, we give a characterization of weighted Hardy spaces $H_{\mathscr{L}}^{1}(\omega)$ via Riesz transforms associated with Schrödinger operator $\mathscr{L}$, where $\mathscr{L}=-\Delta+V$ is a Schrödinger operator on $\mathbb{R}^{n}(n \geq 3)$ and $V$ is a nonnegative function satisfying the reverse Hölder inequality.

\section{Introduction}

Let $\mathscr{L}=-\Delta+V$ be a Schrödinger operator on $\mathbb{R}^{n}, n \geq 3$, where $V \not \equiv 0$ is a fixed nonnegative potential. We assume that $V$ belongs to the reverse Hölder class $R H_{s}\left(\mathbb{R}^{n}\right)$ for some $s \geq n / 2$; that is, there exists $C=C(s, V)>0$ such that

$$
\left(\frac{1}{|B|} \int_{B} V(x)^{s} d x\right)^{1 / s} \leq C\left(\frac{1}{|B|} \int_{B} V(x) d x\right)
$$

for every ball $B \subset \mathbb{R}^{n}$. With regard to the Schrödinger operator $\mathscr{L}$, we know that the operators derived from $\mathscr{L}$ behave "locally" quite similar to those corresponding to the Laplacian (see $[1,2])$. The notion of locality is given by the critical radius function

$$
\rho(x)=\frac{1}{m_{V}(x)}=\sup _{r>0}\left\{r: \frac{1}{r^{n-2}} \int_{B(x, r)} V(y) d y \leq 1\right\} .
$$

Throughout the paper we assume that $V \not \equiv 0$, so that $0<$ $\rho(x)<\infty$ (see [2]).

The study of Schrödinger operator $\mathscr{L}=-\Delta+V$ recently attracted much attention; see [2-14]. In particularly, Dziubański and Zienkiewicz [5, 6] studied Hardy space $H_{\mathscr{L}}^{1}$ associated with Schrödinger operators $\mathscr{L}$. In [8], we introduce the weighted Hardy space $H_{\mathscr{L}}^{1}(\omega)$ with $\omega \in A_{1}\left(\mathbb{R}^{n}\right)$ (for Muckenhoupt's weights, see [15-19]), and in [14], we obtain some weighted estimates for bilinear operators on $H_{\mathscr{L}}^{1}(\omega)$.

Recently, Bongioanni et al. [3] introduced new classes of weights, related to Schrödinger operators $\mathscr{L}$, that is, $A_{p}^{\rho, \infty}\left(\mathbb{R}^{n}\right)$ weight, which are in general larger than Muckenhoupt's (see Section 2 for notions of $A_{p}^{\rho, \infty}\left(\mathbb{R}^{n}\right)$ weight). In $[9,10,20]$, Tang gave some results about weighted norm inequalities with weights $\omega \in A_{p}^{\rho, \infty}\left(\mathbb{R}^{n}\right)$. In [21], we have obtained an atomic characterization for weighted Hardy space $H_{\mathscr{L}}^{1}(\omega)$ with $\omega \in A_{1}^{\rho, \infty}\left(\mathbb{R}^{n}\right)$, by introducing the weighted local Hardy spaces $h_{\rho}^{p}(\omega)$ with $A_{q}^{\rho, \infty}\left(\mathbb{R}^{n}\right)$ weights and establishing the atomic characterization of the weighted local Hardy spaces $h_{\rho}^{p}(\omega)$ with $\omega \in A_{q}^{\rho, \infty}\left(\mathbb{R}^{n}\right)$ weights.

An interesting question is whether we can give a characterization of weighted Hardy spaces $H_{\mathscr{L}}^{1}(\omega)$ with $\omega \in$ $A_{1}^{\rho, \infty}\left(\mathbb{R}^{n}\right)$ via Riesz transforms associated with Schrödinger operator $\mathscr{L}$. Since it is difficult to give the characterization by the same method in $[5,8]$, in this paper, we will establish the characterization by a new method, which will take advantage of weighted local Hardy spaces $h_{\rho}^{p}(\omega)$ and localized Riesz transforms. In fact, by this method, we can give the Riesz transforms characterization of $h_{\rho}^{p}(\omega)$ at the same time.

The paper is organized as follows. In Section 2, we review some notions and notations concerning the weight classes $A_{p}^{\rho, \theta}\left(\mathbb{R}^{n}\right)$ introduced in $[3,9,10]$, and we also review some 
results about $h_{\rho}^{1}(\omega)$ and $H_{\mathscr{L}}^{1}(\omega)$. In Section 3, we establish the Riesz transforms characterization of weighted Hardy spaces $H_{\mathscr{L}}^{1}(\omega)$.

Throughout this paper, we let $C$ denote constants that are independent of the main parameters involved but whose value may differ from line to line. By $A \sim B$, we mean that there exists a constant $C>1$ such that $1 / C \leq A / B \leq C$. The symbol $A \lesssim B$ means that $A \leq C B$. The symbol [s] for $s \in \mathbb{R}$ denotes the maximal integer not more than $s$. We also set $\mathbb{N} \equiv\{1,2, \ldots\}$ and $\mathbb{Z}_{+} \equiv \mathbb{N} \cup\{0\}$.

\section{Preliminaries}

In this section, we first review some notions and notations concerning the weight classes $A_{p}^{\rho, \theta}\left(\mathbb{R}^{n}\right)$ introduced in $[3,9$, 10]. Given $B=B(x, r)$ and $\lambda>0$, we will write $\lambda B$ for the $\lambda$-dilate ball, which is the ball with the same center $x$ and with radius $\lambda r$. Similarly, $Q(x, r)$ denotes the cube centered at $x$ with side length $r$ (here and below only cubes with sides parallel to the axes are considered), and $\lambda Q(x, r)=Q(x, \lambda r)$. Particularly, we will denote $2 B$ by $B^{*}$ and $2 Q$ by $Q^{*}$. The following lemma is a basic property of the critical radius function $\rho(x)$.

Lemma 1 (see [2]). There exist $C_{0} \geq 1$ and $k_{0} \geq 1$ so that for all $x, y \in \mathbb{R}^{n}$

$$
\begin{gathered}
C_{0}^{-1} \rho(x)\left(1+\frac{|x-y|}{\rho(x)}\right)^{-k_{0}} \leq \rho(y) \\
\leq C_{0} \rho(x)\left(1+\frac{|x-y|}{\rho(x)}\right)^{k_{0} /\left(k_{0}+1\right)} .
\end{gathered}
$$

In particular, $\rho(x) \sim \rho(y)$ when $y \in B(x, r)$ and $r \leq C \rho(x)$, where $C$ is a positive constant.

Now we recall the covering of $\mathbb{R}^{n}$ and the partition of unity related to $\rho$ from [5]. For $m \in \mathbb{Z}$ we defined the sets $\mathscr{B}_{m}$ by $\mathscr{B}_{m}=\left\{x: 2^{-(m+1) / 2}<\rho(x) \leq 2^{-m / 2}\right\}$, where critical radii $\rho(x)$ have been defined in (3). Since $0<\rho(x)<\infty$, we have $\mathbb{R}^{n}=\bigcup_{m \in \mathbb{Z}} \mathscr{B}_{m}$.

Lemma 2 (see [5]). There is a positive constant $C$ and $a$ collection of balls $B_{(m, k)}=B\left(x_{(m, k)}, 2^{2-m / 2}\right), m \in \mathbb{Z}, k=$ $1,2, \ldots$, such that $x_{(m, k)} \in \mathscr{B}_{m}, \mathscr{B}_{m} \subset \bigcup_{k} B\left(x_{(m, k)}, 2^{-m / 2}\right)$, and for every $(m, k)$ and $R \geq 2$

$$
\begin{aligned}
& \#\left\{\left(m^{\prime}, k^{\prime}\right): B\left(x_{(m, k)}, R 2^{-m / 2}\right) \cap B\left(x_{\left(m^{\prime}, k^{\prime}\right)}, R 2^{-m^{\prime} / 2}\right)\right. \\
& \quad \neq \emptyset\} \leq R^{C} ;
\end{aligned}
$$

here and in what follows, for any set $E$, \#E denotes its cardinality.

Lemma 3 (see [5]). There are nonnegative functions $\psi_{(m, k)}$ such that

(i) for all $m \in \mathbb{Z}$ and $k \in \mathbb{N}, \psi_{(m, k)} \in C_{c}^{\infty}\left(B\left(x_{(m, k)}\right.\right.$, $\left.2^{1-m / 2}\right)$; (ii) $\sum_{(m, k)} \psi_{(m, k)}(x)=1$ for all $x \in \mathbb{R}^{n}$;

(iii) there exists a positive constant $C$ s.t. for all $m \in \mathbb{Z}, k \in$ $\mathbb{N}$, and $\left\|\nabla \psi_{(m, k)}\right\|_{L^{\infty}\left(\mathbb{R}^{n}\right)} \leq C 2^{m}$.

In this paper, we write $\Psi_{\theta}(B)=\left(1+r / \rho\left(x_{0}\right)\right)^{\theta}$, where $\theta \geq 0$ and $x_{0}$ and $r$ denote the center and radius of $B$, respectively. As in [3], we say that a weight $\omega$ belongs to the class $A_{p}^{\rho, \theta}\left(\mathbb{R}^{n}\right)$ for $1<p<\infty$, if there is a constant $C$ such that for all balls $B$

$$
\begin{aligned}
& \left(\frac{1}{\Psi_{\theta}(B)|B|} \int_{B} \omega(y) d y\right) \\
& \quad \cdot\left(\frac{1}{\Psi_{\theta}(B)|B|} \int_{B} \omega^{-1 /(p-1)}(y) d y\right)^{p-1} \leq C .
\end{aligned}
$$

We also say that a nonnegative function $\omega$ satisfies the $A_{1}^{\rho, \theta}\left(\mathbb{R}^{n}\right)$ condition if there exists a constant $C$ such that $M_{V, \theta}(\omega)(x) \leq C \omega(x)$, a.e. $x \in \mathbb{R}^{n}$, where

$$
M_{V, \theta} f(x) \equiv \sup _{x \in B} \frac{1}{\Psi_{\theta}(B)|B|} \int_{B}|f(y)| d y .
$$

When $V=0$, we denote $M_{0} f(x)$ by $M f(x)$ (the standard Hardy-Littlewood maximal function). It is easy to see that $|f(x)| \leq M_{V, \theta} f(x) \leq M f(x)$ for a.e. $x \in \mathbb{R}^{n}$ and any $\theta \geq 0$. Clearly, the classes $A_{p}^{\rho, \theta}$ are increasing with $\theta$, and we denote $A_{p}^{\rho, \infty}=\bigcup_{\theta \geq 0} A_{p}^{\rho, \theta}$ and $A_{\infty}^{\rho, \infty}=\bigcup_{p \geq 1} A_{p}^{\rho, \infty}$. In addition, for $1 \leq p \leq \infty$, denote by $p^{\prime}$ the adjoint number of $p$; that is, $1 / p+1 / p^{\prime}=1$.

We remark that balls can be replaced by cubes in definition of $A_{p}^{\rho, \theta}$ and $M_{V, \theta}$, since $\Psi(B) \leq \Psi(2 B) \leq 2^{\theta} \Psi(B)$. In fact, for the cube $Q=Q\left(x_{0}, r\right)$, we can also define $\Psi_{\theta}(Q)=$ $\left(1+r / \rho\left(x_{0}\right)\right)^{\theta}$. Now, we give some properties of weights class $A_{p}^{\rho, \theta}$ for $p \geq 1$.

Lemma 4 (see $[3,10,21])$. Let $\omega \in A_{p}^{\rho, \infty}=\bigcup_{\theta \geq 0} A_{p}^{\rho, \theta}$ for $p \geq$ 1. Then

(i) if $1 \leq p_{1}<p_{2}<\infty$, then $A_{p_{1}}^{\rho, \theta} \subset A_{p_{2}}^{\rho, \theta}$;

(ii) if $\omega \in A_{p}^{\rho, \infty}, 1<p<\infty$, then there exists $\epsilon>0$ such that $\omega \in A_{p-\epsilon}^{\rho, \infty}$;

(iii) for $\omega \in A_{p}^{\rho, \theta}, Q=Q(x, r)$, and $\lambda>1$, there exists a positive constant $C$ such that $\omega(\lambda Q) \leq$ $C\left(\Psi_{\theta}(\lambda Q)\right)^{p} \lambda^{n p} \omega(Q)$.

For any $\omega \in A_{\infty}^{\rho, \infty}\left(\mathbb{R}^{n}\right)$, define the critical index of $\omega$ by

$$
q_{\omega} \equiv \inf \left\{p \in[1, \infty): \omega \in A_{p}^{\rho, \infty}\left(\mathbb{R}^{n}\right)\right\} .
$$

For any $\varphi \in \mathscr{D}\left(\mathbb{R}^{n}\right)$, let $\varphi_{t}(x)=t^{-n} \varphi(x / t)$ for $t>0$ and $\varphi_{j}(x)=2^{j n} \varphi\left(2^{j} x\right)$ for $j \in \mathbb{Z}$. Now let us introduce the space $H_{\mathscr{L}}^{1}(\omega)$. Let $\left\{T_{t}\right\}_{t>0}$ be the semigroup of linear operators generated by $\mathscr{L}$ and let $T_{t}(x, y)$ be their kernels; that is, $T_{t} f(x)=e^{-t \mathscr{L}} f(x)=\int_{\mathbb{R}^{n}} T_{t}(x, y) f(y) d y$, for $t>0$ and $f \in L^{2}\left(\mathbb{R}^{n}\right)$. A weighted Hardy-type space related to $\mathscr{L}$ with 
$A_{1}^{\rho, \theta}\left(\mathbb{R}^{n}\right)$ weights is naturally defined by $H_{\mathscr{L}}^{1}(\omega) \equiv\{f \in$ $\left.L_{\omega}^{1}\left(\mathbb{R}^{n}\right): \mathscr{T}^{*} f(x) \in L_{\omega}^{1}\left(\mathbb{R}^{n}\right)\right\}$, with $\|f\|_{H_{f}^{1}(\omega)} \equiv\left\|\mathscr{T}^{*} f\right\|_{L_{\omega}^{1}\left(\mathbb{R}^{n}\right)}$. $H_{\mathscr{L}}^{1}(\omega)$ with $\omega \in A_{1}\left(\mathbb{R}^{n}\right)$ has been studied in $[8,14]$, and we have the following theorem.

Theorem A (see [8]). If $\omega \in A_{1}\left(\mathbb{R}^{n}\right)$ and $V \in R H_{n}\left(\mathbb{R}^{n}\right)$ is a nonnegative potential, then, for all $f \in H_{\mathscr{L}}^{1}(\omega)$, one has $\|f\|_{H_{f}^{1}(\omega)} \sim\|f\|_{L_{\omega}^{1}\left(\mathbb{R}^{n}\right)}+\sum_{j=1}^{n}\left\|R_{j} f\right\|_{L_{\omega}^{1}\left(\mathbb{R}^{n}\right)}$.

Then let us recall some results about weighted local Hardy spaces $h_{\rho}^{p}(\omega)$. We first introduce some local maximal functions. For $N \in \mathbb{Z}_{+}$and $R \in(0, \infty)$, let

$$
\begin{aligned}
& \mathscr{D}_{N, R}\left(\mathbb{R}^{n}\right) \equiv\left\{\varphi \in \mathscr{D}\left(\mathbb{R}^{n}\right): \operatorname{supp}(\varphi)\right. \\
& \quad \subset B(0, R),\|\varphi\|_{\mathscr{D}_{N}\left(\mathbb{R}^{n}\right)} \equiv \sup _{x \in \mathbb{R}^{n}, \alpha \in \mathbb{Z}_{+}^{n},|\alpha| \leq N}\left|\partial^{\alpha} \varphi(x)\right| \\
& \quad \leq 1\} .
\end{aligned}
$$

For any $f \in \mathscr{D}^{\prime}\left(\mathbb{R}^{n}\right)$, the local nontangential grand maximal function $\widetilde{\mathscr{M}}_{N, R}(f)$ of $f$ is defined as

$$
\begin{aligned}
& \widetilde{\mathscr{M}}_{N, R}(f)(x) \equiv \sup \left\{\left|\varphi_{l} * f(z)\right|:|x-z|<2^{-l}\right. \\
& \left.\quad<\rho(x), \varphi \in \mathscr{D}_{N, R}\left(\mathbb{R}^{n}\right)\right\},
\end{aligned}
$$

and the local vertical grand maximal function $\mathscr{M}_{N, R}(f)$ of $f$ is defined as

$$
\begin{aligned}
& \mathscr{M}_{N, R}(f)(x) \equiv \sup \left\{\left|\varphi_{l} * f(x)\right|: 0<2^{-l}<\rho(x), \varphi\right. \\
& \left.\quad \in \mathscr{D}_{N, R}\left(\mathbb{R}^{n}\right)\right\} .
\end{aligned}
$$

For the sake of convenience, we denote $\mathscr{D}_{N, R}\left(\mathbb{R}^{n}\right)$, $\widetilde{\mathscr{M}}_{N, R}(f)$, and $\mathscr{M}_{N, R}(f)$ as $\mathscr{D}_{N}^{0}\left(\mathbb{R}^{n}\right), \widetilde{M}_{N}^{0}(f)$, and $\mathscr{M}_{N}^{0}(f)$, when $R=1$, and as $\mathscr{D}_{N}\left(\mathbb{R}^{n}\right), \widetilde{M}_{N}(f)$, and $\mathscr{M}_{N}(f)$, when $R=\max \left\{R_{1}, R_{2}, R_{3}\right\}>1$ (where $R_{1}, R_{2}$, and $R_{3}$ are defined as in Lemmas 4.2, 4.4, and 4.8 in [21]). For any $N \in \mathbb{Z}_{+}$and $x \in \mathbb{R}^{n}$, obviously, $\mathscr{M}_{N}^{0}(f)(x) \leq \mathscr{M}_{N}(f)(x) \leq \widetilde{\mathscr{M}}_{N}(f)(x)$.

Definition 5. Let $\omega \in A_{\infty}^{\rho, \infty}\left(\mathbb{R}^{n}\right)$ and let $q_{\omega}$ be as in (7), $p \in$ $(0,1]$, and $\widetilde{N}_{p, \omega} \equiv\left[n\left(q_{\omega} / p-1\right)\right]+2$. For each $N \in \mathbb{N}$ with $N \geq$ $\widetilde{N}_{p, \omega}$, the weighted local Hardy space is defined by $h_{\rho, N}^{p}(\omega) \equiv$ $\left\{f \in \mathscr{D}^{\prime}\left(\mathbb{R}^{n}\right): \mathscr{M}_{N}(f) \in L_{\omega}^{p}\left(\mathbb{R}^{n}\right)\right\}$. Moreover, let $\|f\|_{h_{\rho, N}^{p}(\omega)} \equiv$ $\left\|\mathscr{M}_{N}(f)\right\|_{L_{\omega}^{p}\left(\mathbb{R}^{n}\right)}$.

Here and in what follows, we define

$$
N_{p, \omega} \equiv \max \left\{\widetilde{N}_{p, \omega}, N_{0}\right\},
$$

where $\widetilde{N}_{p, \omega}$ and $N_{0}$ are, respectively, as in Definition 2.2 and Theorem 3.1 of [21].
Theorem B (see [21]). Let $\omega \in A_{\infty}^{\rho, \infty}\left(\mathbb{R}^{n}\right)$ and let $N_{p, \omega}$ be as in (11). Then for any integer $N \geq N_{p, \omega}$ and $f \in h_{\rho, N}^{p}(\omega)$, one has

$$
\begin{aligned}
\|f\|_{h_{\rho, N}^{p}(\omega)} & \sim\left\|\widetilde{M}_{N}(f)\right\|_{L_{\omega}^{p}\left(\mathbb{R}^{n}\right)} \sim\left\|\widetilde{\mathscr{M}}_{N}^{0}(f)\right\|_{L_{\omega}^{p}\left(\mathbb{R}^{n}\right)} \\
& \sim\left\|\mathscr{M}_{N}^{0}(f)\right\|_{L_{\omega}^{p}\left(\mathbb{R}^{n}\right)},
\end{aligned}
$$

where the implicit constants are independent of $f$.

Next, we introduce the weighted local atoms and weighted atomic local Hardy space.

Definition 6. Let $\omega \in A_{\infty}^{\rho, \infty}\left(\mathbb{R}^{n}\right)$ and let $q_{\omega}$ be as in (7). A triplet $(p, q, s)_{\omega}$ is called admissible, if $p \in(0,1], q \in\left(q_{\omega}, \infty\right]$, and $s \in \mathbb{N}$ with $s \geq\left[n\left(q_{\omega} / p-1\right)\right]$. A function $a$ on $\mathbb{R}^{n}$ is said to be a $(p, q, s)_{\omega}$-atom if

(i) supp $a \subset Q(x, r)$ and $r \leq L_{1} \rho(x)$,

(ii) $\|a\|_{L_{\omega}^{q}\left(\mathbb{R}^{n}\right)} \leq[\omega(Q)]^{1 / q-1 / p}$,

(iii) $\int_{\mathbb{R}^{n}} a(x) x^{\alpha} d x=0$ for all $\alpha \in \mathbb{Z}_{+}^{n}$ with $|\alpha| \leq s$, when $Q=Q(x, r), r<L_{2} \rho(x)$,

where $L_{1}=4 C_{0}(3 \sqrt{n})^{k_{0}}, L_{2}=1 / C_{0}^{2}(3 \sqrt{n})^{k_{0}+1}$, and $C_{0}$ and $k_{0}$ are constants given in Lemma 1. Moreover, a function $a(x)$ on $\mathbb{R}^{n}$ is called a $(p, q)_{\omega}$-single-atom with $q \in\left(q_{\omega}, \infty\right]$, if $\|a\|_{L_{\omega}^{q}\left(\mathbb{R}^{n}\right)} \leq\left[\omega\left(\mathbb{R}^{n}\right)\right]^{1 / q-1 / p}$.

Lemma 7 (see [21]). Let $\omega \in A_{q}^{\rho, \theta}\left(\mathbb{R}^{n}\right)$ and a be a $(p, q, s)_{\omega^{-}}$ atom, which satisfies supp $a \subset Q\left(x_{0}, r\right)$; then there exists a constant $C$ such that $\|a\|_{L^{1}\left(\mathbb{R}^{n}\right)} \leq C|Q| \omega(Q)^{-1 / p} \Psi_{\theta}(Q)$.

Definition 8. Let $\omega \in A_{\infty}^{\rho, \infty}\left(\mathbb{R}^{n}\right)$ and let $q_{\omega}$ be as in (7), and let $(p, q, s)_{\omega}$ be admissible. The weighted atomic local Hardy space $h_{\rho}^{p, q, s}(\omega)$ is defined as the set of all $f \in \mathscr{D}^{\prime}\left(\mathbb{R}^{n}\right)$ satisfying that $f=\sum_{i=0}^{\infty} \lambda_{i} a_{i}$ in $\mathscr{D}^{\prime}\left(\mathbb{R}^{n}\right)$, where $\left\{a_{i}\right\}_{i \in \mathbb{N}}$ are $(p, q, s)_{\omega}$-atoms, $a_{0}$ is a $(p, q)_{\omega}$-single-atom, and $\left\{\lambda_{i}\right\}_{i \in \mathbb{Z}_{+}} \subset \mathbb{C}$. Moreover, the quasi-norm of $f \in h_{\rho}^{p, q, s}(\omega)$ is defined by $\|f\|_{h_{\rho}^{p, q, s}(\omega)} \equiv \inf \left\{\left[\sum_{i=0}^{\infty}\left|\lambda_{i}\right|^{p}\right]^{1 / p}\right\}$, where the infimum is taken over all the decompositions of $f$ as above.

Theorem C (see [21]). Let $\omega \in A_{\infty}^{\rho, \infty}\left(\mathbb{R}^{n}\right)$, and let $q_{\omega}$ and $N_{p, \omega}$ be, respectively, as in (7) and (11). If $q \in\left(q_{\omega}, \infty\right], p \in(0,1]$ and integers $s$ and $N$ satisfy $N \geq N_{p, \omega}$ and $N>s \geq\left[n\left(q_{\omega} / p-1\right)\right]$, then $h_{\rho}^{p, q, s}(\omega)=h_{\rho, N}^{p}(\omega)=h_{\rho, N_{p, \omega}^{p}}^{p}(\omega)$ with equivalent norms.

In virtue of the above theorem, for notational simplicity, we denote by $h_{\rho}^{p}(\omega)$ the weighted local Hardy space $h_{\rho, N}^{p}(\omega)$ when $N \geq N_{p, \omega}$.

Definition 9. Let $\omega \in A_{\infty}^{\rho, \infty}\left(\mathbb{R}^{n}\right)$ and let $(p, q, s)_{\omega}$ be admissible; then $h_{\rho, \text { fin }}^{p, q, s}(\omega)$ is defined as the vector space of all finite linear combinations of $(p, q, s)_{\omega}$-atoms and a $(p, q)_{\omega}$-singleatom, and the norm of $f$ in $h_{\rho, \text { fin }}^{p, q, s}(\omega)$ is defined by $\|f\|_{h_{\rho, \text { fin }}^{p, q, s}(\omega)} \equiv$ $\inf \left\{\left[\sum_{i=0}^{k}\left|\lambda_{i}\right|^{p}\right]^{1 / p}\right\}$, where $f=\sum_{i=0}^{k} \lambda_{i} a_{i}, k \in \mathbb{Z}_{+},\left\{\lambda_{i}\right\}_{i=0}^{k, \text { in }} \subset \mathbb{C}$, $\left\{a_{i}\right\}_{i=1}^{k}$ are $(p, q, s)_{\omega}$-atoms, and $a_{0}$ is a $(p, q)_{\omega}$-single-atom. 
Let $\beta \in(0,1]$. A quasi-Banach space $\mathscr{B}_{\beta}$ with the quasinorm $\|\cdot\|_{\mathscr{B}_{\beta}}$ is called a $\beta$-quasi-Banach space if $\|f+g\|_{\mathscr{B}_{\beta}}^{\beta} \leq$ $\|f\|_{\mathscr{B}_{\beta}}^{\beta}+\|g\|_{\mathscr{B}_{\beta}}^{\beta}$ for all $f, g \in \mathscr{B}_{\beta}$. For any given $\beta$-quasiBanach space $\mathscr{B}_{\beta}$ with $\beta \in(0,1]$ and a linear space $\mathscr{Y}$, an operator $T$ from $\mathcal{Y}$ to $\mathscr{B}_{\beta}$ is said to be $\mathscr{B}_{\beta}$-sublinear if, for any $f, g \in \mathscr{B}_{\beta}$ and $\lambda, v \in \mathbb{C},\|T(\lambda f+v g)\|_{\mathscr{B}_{\beta}} \leq\left(|\lambda|^{\beta}\|T(f)\|_{\mathscr{B}_{\beta}}^{\beta}+\right.$ $\left.|\nu|^{\beta}\|T(g)\|_{\mathscr{B}_{\beta}}^{\beta}\right)^{1 / \beta}$ and $\|T(f)-T(g)\|_{\mathscr{B}_{\beta}} \leq\|T(f-g)\|_{\mathscr{B}_{\beta}}$.

Theorem D (see [21]). Let $\omega \in A_{\infty}^{\rho, \infty}\left(\mathbb{R}^{n}\right), 0<p \leq$ $\beta \leq 1$, and $\mathscr{B}_{\beta}$ be a $\beta$-quasi-Banach space. Suppose $q \in\left(q_{\omega}, \infty\right)$ and $T: h_{\rho, \text { fin }}^{p, q, s}(\omega) \rightarrow \mathscr{B}_{\beta}$ is a $\mathscr{B}_{\beta}$-sublinear operator such that $S \equiv \sup \left\{\|T(a)\|_{\mathscr{B}_{\beta}}\right.$ : $a$ is a $(p, q, s)_{\omega}$-atom or $(p, q)_{\omega}$ single-atom $\}<\infty$. Then there exists a unique bounded $\mathscr{B}_{\beta}$-sublinear operator $\widetilde{T}$ from $h_{\rho}^{p}(\omega)$ to $\mathscr{B}_{\beta}$ which extends T.

Theorem E (see [21]). Let $0 \not \equiv V \in R H_{n / 2}$ and $\omega \in A_{1}^{\rho, \infty}\left(\mathbb{R}^{n}\right)$; then $h_{\rho}^{1}(\omega)=H_{\mathscr{L}}^{1}(\omega)$ with equivalent norms; that is, $\|f\|_{h_{\rho}^{1}(\omega)} \sim$ $\|f\|_{H_{\mathscr{f}}^{1}(\omega)}$.

\section{Riesz Transform Characterization of Weighted Hardy Spaces}

The main purpose of this section is to give a characterization of weighted Hardy spaces $H_{\mathscr{L}}^{1}(\omega)$ with $\omega \in A_{1}^{\rho, \infty}\left(\mathbb{R}^{n}\right)$ via Riesz transforms associated with Schrödinger operator $\mathscr{L}$. We begin with some useful lemmas.

Lemma 10 (see [8]). If $\omega \in A_{1}\left(\mathbb{R}^{n}\right)$, then there is a constant $C>0$ such that

$$
\sum_{(m, k)}\left\|R_{j}\left(\psi_{(m, k)} f\right)-\psi_{(m, k)} R_{j}(f)\right\|_{L^{1}(\omega)} \leq C\|f\|_{L^{1}(\omega)} .
$$

Lemma 11 (see [5]). There exists a sequence of points $x_{j} \in \mathbb{R}^{n}$, $j \geq 1$, such that the family $B_{j}=B\left(x_{j}, \rho\left(x_{j}\right)\right), j \geq 1$, satisfies the following:

(a) $\bigcup_{j} B_{j}=\mathbb{R}^{n}$.

(b) For every $\sigma \geq 1$ there exist constants $C$ and $N_{1}$ such that $\Sigma_{j} \chi_{\sigma B_{j}} \leq C \sigma^{N_{1}}$.

For $j=1,2, \ldots, n$, let us define the Riesz transforms $R_{j}$ associated with Schrödinger operator $\mathscr{L}$ by $R_{j}=$ $\left(\partial / \partial x_{j}\right) \mathscr{L}^{-1 / 2}$. In addition, as in [22], for all $j \in\{1,2, \ldots, n\}$ and $x \in \mathbb{R}^{n}$, we define localized Riesz transforms as

$$
\begin{aligned}
& R_{j}^{l}(f)(x) \\
& \quad \equiv \text { p.v. } c_{n} \int_{\mathbb{R}^{n}} \frac{x_{j}-y_{j}}{|x-y|^{n+1}} \eta\left(\frac{|x-y|}{\rho(x)}\right) f(y) d y,
\end{aligned}
$$

where and in what follows $c_{n} \equiv \Gamma((n+1) / 2) /\left[\pi^{(n+1) / 2}\right], \eta \in$ $C^{1}\left(\mathbb{R}^{n}\right)$ supported in $(-1,1)$, and $\eta(t)=1$ if $|t| \leq 1 / 2$. For all $j \in\{1,2, \ldots, n\}$ and $x \in \mathbb{R}^{n}$, we define

$$
R_{j, *}(f)(x) \equiv c_{n} \sup _{\varepsilon>0}\left|\int_{|x-y| \geq \varepsilon} \frac{x_{j}-y_{j}}{|x-y|^{n+1}} f(y) d y\right| .
$$

With these operators, we can get the following boundedness.

Lemma 12. Let $R_{j}^{l}$ be as in (14); then

(i) $\left\|R_{j}^{l}(f)\right\|_{L_{\omega}^{p}\left(\mathbb{R}^{n}\right)} \leq C_{p, \omega}\|f\|_{L_{\omega}^{p}\left(\mathbb{R}^{n}\right)}$, for $1<p<\infty$ and $\omega \in A_{p}\left(\mathbb{R}^{n}\right)$,

(ii) $\left\|R_{j}^{l}(f)\right\|_{L_{\omega}^{1, \infty}\left(\mathbb{R}^{n}\right)} \leq C_{\omega}\|f\|_{L_{\omega}^{1}\left(\mathbb{R}^{n}\right)}$, for $\omega \in A_{1}\left(\mathbb{R}^{n}\right)$.

Proof. Let $j \in\{1,2, \ldots, n\}$; then by $[15,18]$, we know that $R_{j, *}$ is bounded on $L_{\omega}^{p}\left(\mathbb{R}^{n}\right)$ for $1<p<\infty$ and bounded from $L_{\omega}^{1}\left(\mathbb{R}^{n}\right)$ to $L_{\omega}^{1, \infty}\left(\mathbb{R}^{n}\right)$. Let $\left\{\widetilde{R}_{j}\right\}_{j=1}^{n}$ be the classical Riesz transforms; then for all $x \in \mathbb{R}^{n}$, we have

$$
\begin{aligned}
& \left|R_{j}^{l}(f)(x)-\widetilde{R}_{j}(f)(x)\right| \\
& \quad \leq \mid \int_{(1 / 2) \rho(x) \leq|x-y|<\rho(x)} \frac{x_{j}-y_{j}}{|x-y|^{n+1}}[1 \\
& \left.\quad-\eta\left(\frac{|x-y|}{\rho(x)}\right)\right] f(y) d y \mid \\
& \quad+\left|\int_{|x-y| \geq \rho(x)} \frac{x_{j}-y_{j}}{|x-y|^{n+1}} f(y) d y\right| \lesssim M(f)(x) \\
& \quad+R_{j, *}(f)(x),
\end{aligned}
$$

where and in what follows $M$ denotes the Hardy-Littlewood maximal operator. By the above estimate, combining with the boundedness on $L_{\omega}^{p}\left(\mathbb{R}^{n}\right)$ for $1<p<\infty$ and boundedness from $L_{\omega}^{1}\left(\mathbb{R}^{n}\right)$ to $L_{\omega}^{1, \infty}\left(\mathbb{R}^{n}\right)$ of $M, \widetilde{R}_{j}$, and $R_{j, *}$, we can obtain the conclusions of the lemma. lemma.

Then for $\omega \in A_{\infty}^{\rho, \infty}\left(\mathbb{R}^{n}\right)$, we can obtain the following

Lemma 13. Let $R_{j}^{l}$ be as in (14); then

(i) $\left\|R_{j}^{l}(f)\right\|_{L_{\omega}^{p}\left(\mathbb{R}^{n}\right)} \leq C_{p, \omega}\|f\|_{L_{\omega}^{p}\left(\mathbb{R}^{n}\right)}$, for $1<p<\infty$ and $\omega \in A_{p}^{\rho, \infty}\left(\mathbb{R}^{n}\right)$,

(ii) $\left\|R_{j}^{l}(f)\right\|_{L_{\omega}^{1, \infty}\left(\mathbb{R}^{n}\right)} \leq C_{\omega}\|f\|_{L_{\omega}^{1}\left(\mathbb{R}^{n}\right)}$, for $\omega \in A_{1}^{\rho, \infty}\left(\mathbb{R}^{n}\right)$.

Proof. By Lemma 11, there exists a sequence of points $x_{j} \in \mathbb{R}^{n}$, $j \geq 1$, such that $\bigcup_{j} B_{j}=\bigcup_{j} B\left(x_{j}, \rho\left(x_{j}\right)\right)=\mathbb{R}^{n}$. Let $\sigma_{1}=$ $C_{0} 2^{k_{0} /\left(k_{0}+1\right)}+1$, with $C_{0}, k_{0}$ as in Lemma 1 , and set $\widetilde{B}_{j}=\sigma_{1} B_{j}$. Then by Lemma 1 of [3], for $\omega \in A_{p}^{\rho, \infty}\left(\mathbb{R}^{n}\right)$ and any $B_{j}$, there 
exists $\bar{\omega} \in A_{p}\left(\mathbb{R}^{n}\right)$ such that $\bar{\omega}=\omega$ on $\widetilde{B}_{j}$. Thus, by Lemma 12 , we have

$$
\begin{aligned}
\left\|R_{j}^{l}(f)\right\|_{L_{\omega}^{p}\left(B_{j}\right)} & =\left\|R_{j}^{l}\left(\chi_{\widetilde{B}_{j}} f\right)\right\|_{L_{\omega}^{p}\left(B_{j}\right)} \\
& \lesssim\left\|R_{j}^{l}\left(\chi_{\widetilde{B}_{j}} f\right)\right\|_{L_{\bar{\omega}}^{p}\left(B_{j}\right)} \lesssim\left\|\chi_{\widetilde{B}_{j}} f\right\|_{L_{\bar{\omega}}^{p}\left(\mathbb{R}^{n}\right)} \\
& \lesssim\|f\|_{L_{\omega}^{p}\left(\widetilde{B}_{j}\right)} .
\end{aligned}
$$

Summing up all balls of $\left\{B_{j}\right\}_{j}$ gives (i).

For (ii), as in (i), for $\omega \in A_{1}^{\rho, \infty}\left(\mathbb{R}^{n}\right)$ and any $B_{j}$, there exists $\bar{\omega} \in A_{1}\left(\mathbb{R}^{n}\right)$ such that $\bar{\omega}=\omega$ on $\widetilde{B}_{j}$. Then, by Lemma 12 , we have

$$
\begin{aligned}
\omega( & \left.\left\{x \in B_{j}:\left|R_{j}^{l}(f)(x)\right|>\lambda\right\}\right) \\
& \leq \omega\left(\left\{x \in B_{j}:\left|R_{j}^{l}\left(\chi_{\widetilde{B}_{j}} f\right)(x)\right|>\lambda\right\}\right) \\
& =\bar{\omega}\left(\left\{x \in B_{j}:\left|R_{j}^{l}\left(\chi_{\widetilde{B}_{j}} f\right)(x)\right|>\lambda\right\}\right) \\
& \leq \lambda^{-1}\left\|\chi_{\widetilde{B}_{j}} f\right\|_{L_{\bar{\omega}}^{1}\left(\mathbb{R}^{n}\right)} \leq \lambda^{-1}\|f\|_{L_{\omega}^{1}\left(\widetilde{B}_{j}\right)} .
\end{aligned}
$$

Summing up all balls of $\left\{B_{j}\right\}_{j}$ gives (ii).

The proof is complete.

Now we can establish the Riesz transform characterization as follows.

Theorem 14. Let $\omega \in A_{1}^{\rho, \infty}\left(\mathbb{R}^{n}\right)$ and $V \in R H_{n}\left(\mathbb{R}^{n}\right)$; then one has

$$
\begin{aligned}
\|f\|_{H_{\mathscr{L}}^{1}(\omega)} & \sim\|f\|_{h_{\rho}^{1}(\omega)} \sim\|f\|_{L_{\omega}^{1}\left(\mathbb{R}^{n}\right)}+\sum_{j=1}^{n}\left\|R_{j}^{l}(f)\right\|_{L_{\omega}^{1}\left(\mathbb{R}^{n}\right)} \\
& \sim\|f\|_{L_{\omega}^{1}\left(\mathbb{R}^{n}\right)}+\sum_{j=1}^{n}\left\|R_{j}(f)\right\|_{L_{\omega}^{1}\left(\mathbb{R}^{n}\right)} .
\end{aligned}
$$

Proof. $\|f\|_{H_{\mathscr{L}}^{1}(\omega)} \sim\|f\|_{h_{\rho}^{1}(\omega)}$ is obvious by Theorem E. We now prove

$$
\|f\|_{h_{\rho}^{1}(\omega)} \sim\|f\|_{L_{\omega}^{1}\left(\mathbb{R}^{n}\right)}+\sum_{j=1}^{n}\left\|R_{j}^{l}(f)\right\|_{L_{\omega}^{1}\left(\mathbb{R}^{n}\right)} .
$$

We first assume that

$$
\|f\|_{L_{\omega}^{1}\left(\mathbb{R}^{n}\right)}+\sum_{j=1}^{n}\left\|R_{j}^{l} f\right\|_{L_{\omega}^{1}\left(\mathbb{R}^{n}\right)}<\infty
$$

and here we will borrow some idea from [23]. Let $B_{(m, k)}$ be a ball as in Lemma 2, and let $\zeta_{a_{1} B_{(m, k)}}$ be a $C_{0}^{\infty}$ nonnegative function supported in $2 a_{1} B_{(m, k)}$ and $\zeta_{a_{1} B_{(m, k)}}=1$ on $a_{1} B_{(m, k)}$, where $a_{1}=3 C_{0}+1$. By properties of weights, we can set $\bar{\omega} \in A_{1}\left(\mathbb{R}^{n}\right)$ so that $\bar{\omega}=\omega$ on $a_{5} B_{(m, k)}$, where $a_{5}$ is a constant independent of $B_{(m, k)}$ and will be given in the following proof. Taking $\varphi \in \mathscr{D}_{N}^{0}\left(\mathbb{R}^{n}\right)$, by Theorem A, we have

$$
\begin{aligned}
& \left\|\sup _{0<2^{-l}<\rho(x)}\left|\varphi_{l} * f\right|\right\| \|_{L_{\omega}^{1}\left(B_{(m, k)}\right)} \\
& \quad \leq\left\|\sup _{0<2^{-l}<\rho(x)}\left|\varphi_{l} *\left(f \zeta_{a_{1} B_{(m, k)}}\right)\right|\right\|_{L_{\bar{\omega}}^{1}\left(\mathbb{R}^{n}\right)} \\
& \quad \leq\left\|f \zeta_{a_{1} B_{(m, k)}}\right\|_{H_{\mathscr{L}}^{1}(\bar{\omega})} \\
& \quad \leq\left\|f \zeta_{a_{1} B_{(m, k)}}\right\|_{L_{\bar{\omega}}^{1}\left(\mathbb{R}^{n}\right)}+\sum_{j=1}^{n}\left\|R_{j}\left(f \zeta_{a_{1} B_{(m, k)}}\right)\right\|_{L_{\bar{\omega}}^{1}\left(\mathbb{R}^{n}\right)} .
\end{aligned}
$$

Let us denote the integral kernel of the operator $\left(\partial / \partial x_{j}\right) \mathscr{L}^{-1 / 2}$ by $R_{j}(x, y)$, and let $\widetilde{R}_{j}(x, y)$ be the kernel of the classical Riesz transform $\widetilde{R}_{j} f=\left(\partial / \partial x_{j}\right) \Delta^{-1 / 2} f$. Then we can define

$$
\bar{R}_{j}(f)(x) \equiv \int_{\mathbb{R}^{n}} R_{j}(x, y) \eta\left(\frac{|x-y|}{\rho(x)}\right) f(y) d y,
$$

and we also have

$$
R_{j}^{l}(f)(x)=\int_{\mathbb{R}^{n}} \widetilde{R}_{j}(x, y) \eta\left(\frac{|x-y|}{\rho(x)}\right) f(y) d y .
$$

Let $a_{2}=C_{0}\left(1+16 a_{1}\right)+2 a_{1}<a_{5}$; then we have

$$
\begin{aligned}
& \left\|R_{j}\left(f \zeta_{a_{1} B_{(m, k)}}\right)-\zeta_{a_{1} B_{(m, k)}} R_{j}^{l}(f)\right\|_{L_{\bar{\omega}}^{1}\left(\mathbb{R}^{n}\right)} \\
& \quad \leq \| R_{j}\left(f \zeta_{a_{1} B_{(m, k)}} \chi_{a_{2} B_{(m, k)}}\right) \\
& \quad-\zeta_{a_{1} B_{(m, k)}} R_{j}\left(f \chi_{a_{2} B_{(m, k)}}\right) \|_{L_{\bar{\omega}}^{1}\left(\mathbb{R}^{n}\right)} \\
& +\| \zeta_{a_{1} B_{(m, k)}} R_{j}\left(f \chi_{a_{2} B_{(m, k)}}\right) \\
& \quad-\zeta_{a_{1} B_{(m, k)}} \bar{R}_{j}\left(f \chi_{a_{2} B_{(m, k)}}\right) \|_{L_{\bar{\omega}}^{1}\left(\mathbb{R}^{n}\right)} \\
& +\| \zeta_{a_{1} B_{(m, k)}} \bar{R}_{j}\left(f \chi_{a_{2} B_{(m, k)}}\right) \\
& \quad-\zeta_{a_{1} B_{(m, k)}} R_{j}^{l}\left(f \chi_{a_{2} B_{(m, k)}}\right) \|_{L_{\bar{\omega}}^{1}\left(\mathbb{R}^{n}\right)} \equiv I_{1}+I_{2}+I_{3} .
\end{aligned}
$$

For $I_{1}$, by the same method of the proof of Lemma 10 (Lemma 3.14 of [8]), we get

$$
I_{1} \lesssim\|f\|_{L^{1}\left(a_{2} B_{(m, k)}, \bar{\omega}\right)} \leqslant\|f\|_{L_{\omega}^{1}\left(a_{2} B_{(m, k)}\right)} .
$$


For $I_{2}$, by Lemma 3 of [24], we have

$$
\begin{aligned}
I_{2} & \lesssim \| \zeta_{a_{1} B_{(m, k)}} R_{j}\left(f \chi_{a_{2} B_{(m, k)}}\right)-\left.\zeta_{a_{1} B_{(m, k)}} \bar{R}_{j}\left(f \chi_{\left.a_{2} B_{(m, k)}\right)}\right)\right|_{L_{\bar{\omega}}^{1}\left(\mathbb{R}^{n}\right)} \\
& =\left\|R_{j}\left(f \chi_{a_{2} B_{(m, k)}}\right)-\bar{R}_{j}\left(f \chi_{a_{2} B_{(m, k)}}\right)\right\|_{L^{1}\left(2 a_{1} B_{(m, k)}, \bar{\omega}\right)} \\
& \lesssim \int_{2 a_{1} B_{(m, k)}} \int_{|x-y|>\rho(x) / 2}\left|R_{j}(x, y)\right|\left|1-\eta\left(\frac{|x-y|}{\rho(x)}\right)\right| \\
& \cdot\left|f \chi_{a_{2} B_{(m, k)}}(y)\right| d y \bar{\omega}(x) d x \\
& \leqslant \int_{2 a_{1} B_{(m, k)}} \int_{|x-y|>\rho(x) / 2} \frac{\left|f \chi_{a_{2} B_{(m, k)}}(y)\right|}{|x-y|^{n}} d y \bar{\omega}(x) d x \\
& \lesssim \int_{2 a_{1} B_{(m, k)}} \int_{|x-y|>\rho(x) / 2} \frac{\left|f \chi_{a_{2} B_{(m, k)}}(y)\right|}{(\rho(x))^{n}} d y \bar{\omega}(x) d x .
\end{aligned}
$$

Since $x \in 2 a_{1} B_{(m, k)}$ and $y \in a_{2} B_{(m, k)}$, we have $\rho(x) \sim$ $\rho\left(x_{(m, k)}\right) \sim \rho(y)$, and there is a constant $a_{3}>1$ independent of $B_{(m, k)}$ such that

$$
\begin{aligned}
I_{2} & \lesssim \int_{a_{2} B_{(m, k)}}\left(\int_{|x-y|<a_{3} \rho(y)} \frac{\bar{\omega}(x)}{(\rho(y))^{n}} d x\right) \\
& \cdot\left|f \chi_{a_{2} B_{(m, k)}}(y)\right| d y \lesssim \int_{a_{2} B_{(m, k)}}|f(y)| \bar{\omega}(y) d y \\
& \leqslant\|f\|_{L_{\omega}^{1}\left(a_{2} B_{(m, k)}\right)} .
\end{aligned}
$$

We now estimate $I_{3}$. For $x \in 2 a_{1} B_{(m, k)}$ and $y \in a_{2} B_{(m, k)}$, we have $\rho(x) \sim \rho\left(x_{(m, k)}\right) \sim \rho(y)$, and there exists a constant $a_{4}>$ 1 independent of $B_{(m, k)}$ such that $\rho(x) \leq a_{4} \rho(y)$. On the other hand, if $V \in R H_{n}\left(\mathbb{R}^{n}\right)$, then there exists $\varepsilon>0$, which depends only on $n$ and the constant $C$ in (3), such that $V \in R H_{n+\varepsilon}\left(\mathbb{R}^{n}\right)$ (see [25]). Thus, according to Lemma 3 of [24], we get

$$
\begin{aligned}
I_{3} & \lesssim \int_{2 a_{1} B_{(m, k)}} \int_{\mathbb{R}^{n}}\left|R_{j}(x, y)-\widetilde{R}_{j}(x, y)\right|\left|\eta\left(\frac{|x-y|}{\rho(x)}\right)\right|\left|f \chi_{a_{2} B_{(m, k)}}(y)\right| d y \bar{\omega}(x) d x \lesssim \int_{2 a_{1} B_{(m, k)}} \int_{|x-y|<\rho(x)} \mid R_{j}(x, y) \\
& -\widetilde{R}_{j}(x, y)|| f \chi_{a_{2} B_{(m, k)}}(y) \mid d y \bar{\omega}(x) d x \lesssim \int_{2 a_{1} B_{(m, k)}} \int_{|x-y|<\rho(x)} \frac{\left|f \chi_{a_{2} B_{(m, k)}}(y)\right|}{|x-y|^{n}}\left(\frac{|x-y|}{\rho(x)}\right)^{2-n /(n+\varepsilon)} d y \bar{\omega}(x) d x \\
& \lesssim \int_{a_{2} B_{(m, k)}}\left(\int_{|x-y|<a_{4} \rho(y)} \frac{1}{|x-y|^{n}}\left(\frac{|x-y|}{\rho(y)}\right)^{2-n /(n+\varepsilon)} \bar{\omega}(x) d x\right)|f(y)| d y .
\end{aligned}
$$

For the inner integral, there exists a constant $a_{5}$ independent of $B_{(m, k)}$ such that $a_{5}>a_{2}$ and $B\left(y, \max \left\{a_{3}, 2 a_{4}\right\} \rho(y)\right) \subset$ $a_{5} B_{(m, k)}$ for all $y \in a_{2} B_{(m, k)}$; then by the properties of $A_{1}$ weights, we have

$$
\begin{aligned}
& \int_{|x-y|<a_{4} \rho(y)} \frac{1}{|x-y|^{n}}\left(\frac{|x-y|}{\rho(y)}\right)^{2-n /(n+\varepsilon)} \bar{\omega}(x) d x \\
& \leq \sum_{k=k_{0}}^{\infty} \int_{|x-y| \sim 2^{-k} \rho(y)} \frac{1}{|x-y|^{n}}\left(\frac{|x-y|}{\rho(y)}\right)^{2-n /(n+\varepsilon)} \\
& \cdot \bar{\omega}(x) d x \\
& \leq \sum_{k=k_{0}}^{\infty} \int_{|x-y| \sim 2^{-k} \rho(y)} \frac{\left(2^{-k}\right)^{2-n /(n+\varepsilon)}}{\left|2^{-k} \rho(y)\right|^{n}} \bar{\omega}(x) d x \\
& \quad \lesssim \sum_{k=k_{0}}^{\infty}\left(2^{-k}\right)^{2-n /(n+\varepsilon)} \int_{|x-y| \leq 2^{-k} \rho(y)} \frac{\bar{\omega}(x)}{\left|2^{-k} \rho(y)\right|^{n}} d x \\
& \leq \bar{\omega}(y),
\end{aligned}
$$

where $k_{0}$ satisfies $2^{-k_{0}-1}<a_{5} \leq 2^{-k_{0}}$. Hence, we obtain

$$
I_{3} \lesssim \int_{a_{2} B_{(m, k)}}|f(y)| \bar{\omega}(y) d y \leqslant\|f\|_{L_{\omega}^{1}\left(a_{2} B_{(m, k)}\right)} .
$$

Combining (22), (26), (28), and (31), we get

$$
\begin{aligned}
& \left\|\sup _{0<2^{-l}<\rho(x)}\left|\varphi_{l} * f\right|\right\|_{L_{\omega}^{1}\left(B_{(m, k)}\right)} \\
& \quad \leq\|f\|_{L_{\omega}^{1}\left(a_{2} B_{(m, k)}\right)}+\sum_{j=1}^{n}\left\|R_{j}^{l}(f)\right\|_{L_{\omega}^{1}\left(2 a_{1} B_{(m, k)}\right)} .
\end{aligned}
$$

Therefore, by Theorem B and Lemma 2, we obtain

$$
\begin{aligned}
& \|f\|_{h_{\rho}^{1}(\omega)} \lesssim \sum_{(m, k)}\left\|\sup _{0<2^{-l}<\rho(x)}\left|\varphi_{l} * f\right|\right\|_{L_{\omega}^{1}\left(B_{(m, k)}\right)} \\
& \quad \lesssim \sum_{(m, k)}\left(\|f\|_{L_{\omega}^{1}\left(a_{2} B_{(m, k)}\right)}+\sum_{j=1}^{n}\left\|R_{j}^{l}(f)\right\|_{L_{\omega}^{1}\left(2 a_{1} B_{(m, k)}\right)}\right) \\
& \quad \lesssim\|f\|_{L_{\omega}^{1}\left(\mathbb{R}^{n}\right)}+\sum_{j=1}^{n}\left\|R_{j}^{l}(f)\right\|_{L_{\omega}^{1}\left(\mathbb{R}^{n}\right)} .
\end{aligned}
$$

In order to prove the converse inequality, by Theorem $\mathrm{D}$, it suffices to show that, for all $j$ and any $(1,2, s)_{\omega}$-atom or $(1,2)_{\omega}$-single-atom $a$,

$$
\left\|R_{j}^{l}(a)\right\|_{L_{\omega}^{1}\left(\mathbb{R}^{n}\right)} \lesssim 1
$$


If $a$ is a single atom, by Hölder inequality and $L_{\omega}^{2}\left(\mathbb{R}^{n}\right)$ boundedness of $R_{j}^{l}$, we have

$$
\begin{aligned}
\left\|R_{j}^{l}(a)\right\|_{L_{\omega}^{1}\left(\mathbb{R}^{n}\right)} & \leqslant\left\|R_{j}^{l}(a)\right\|_{L_{\omega}^{2}\left(\mathbb{R}^{n}\right)} \omega\left(\mathbb{R}^{n}\right)^{1 / 2} \\
& \leqslant\|a\|_{L_{\omega}^{2}\left(\mathbb{R}^{n}\right)} \omega\left(\mathbb{R}^{n}\right)^{1 / 2} \lesssim 1 .
\end{aligned}
$$

Next we assume that $a$ is a $(1,2, s)_{\omega}$-atom and $\operatorname{supp} a \quad C$ $Q\left(x_{0}, r\right)$ with $r \leq L_{1} \rho\left(x_{0}\right)$; then $\rho(x) \leq C_{0}^{2} 2^{k_{0}}\left(1+L_{1}\right) \rho\left(x_{0}\right)$ for any $x$ satisfying $|x-y|<\rho(x)$ and $y \in Q\left(x_{0}, r\right)$.

We first consider the atom $a$ with $L_{2} \rho\left(x_{0}\right) \leq r \leq L_{1} \rho\left(x_{0}\right)$. Taking $b_{1}=1+C_{0}^{2} 2^{k_{0}}\left(1+L_{1}\right) / L_{2}$, then $\operatorname{supp}\left(R_{j}^{l}(a)\right) C$ $Q\left(x_{0}, b_{1} r\right)$; thus, by Hölder inequality, Lemma 4 , and $L_{\omega}^{2}\left(\mathbb{R}^{n}\right)$ boundedness of $R_{j}^{l}$, we get

$$
\begin{aligned}
\left\|R_{j}^{l}(a)\right\|_{L_{\omega}^{1}\left(\mathbb{R}^{n}\right)} & =\left\|R_{j}^{l}(a)\right\|_{L_{\omega}^{1}\left(Q\left(x_{0}, b_{1} r\right)\right)} \\
& \lesssim\|a\|_{L_{\omega}^{2}\left(\mathbb{R}^{n}\right)} \omega\left(Q\left(x_{0}, b_{1} r\right)\right)^{1 / 2} \\
& \lesssim\|a\|_{L_{\omega}^{2}\left(\mathbb{R}^{n}\right)} \omega\left(Q\left(x_{0}, r\right)\right)^{1 / 2} \lesssim 1 .
\end{aligned}
$$

If $r<L_{2} \rho\left(x_{0}\right)$, then $\operatorname{supp}\left(R_{j}^{l}(a)\right) \subset Q\left(x_{0}, b_{2} \rho\left(x_{0}\right)\right)$, where $b_{2}=C_{0}^{2} 2^{k_{0}}\left(1+L_{1}\right)+L_{2}$. Applying the Hölder inequality, Lemma 4 and $L_{\omega}^{2}\left(\mathbb{R}^{n}\right)$ boundedness of $R_{j}^{l}$ give us that

$$
\left\|R_{j}^{l}(a)\right\|_{L_{\omega}^{1}\left(\mathrm{Q}\left(x_{0}, 2 r\right)\right)} \leqslant\|a\|_{L_{\omega}^{2}\left(\mathbb{R}^{n}\right)} \omega\left(Q\left(x_{0}, r\right)\right)^{1 / 2} \leqslant 1 .
$$

Moreover, for $x \in Q\left(x_{0}, b_{2} \rho\left(x_{0}\right)\right) \backslash Q\left(x_{0}, 2 r\right)$, there is a constant $b_{3}>1$ such that $b_{3}^{-1} \rho\left(x_{0}\right) \leq \rho(x) \leq b_{3} \rho\left(x_{0}\right)$, and by the vanishing moment of $a$, we get

$$
\begin{aligned}
& \left|R_{j}^{l}(a)(x)\right| \\
& \leq\left|\int_{\mathbb{R}^{n}} \frac{x_{j}-y_{j}}{|x-y|^{n+1}}\left[\eta\left(\frac{|x-y|}{\rho(x)}\right)-1\right] a(y) d y\right| \\
& \quad+\left|\int_{\mathbb{R}^{n}}\left[\frac{x_{j}-y_{j}}{|x-y|^{n+1}}-\frac{x_{j}-\left(x_{0}\right)_{j}}{\left|x-x_{0}\right|^{n+1}}\right] a(y) d y\right| \\
& \leq \int_{|x-y| \geq \rho\left(x_{0}\right) / 2 b_{3}} \frac{|a(y)|}{|x-y|^{n}} d y \\
& \quad+\int_{\mathbb{R}^{n}} \frac{\left|x_{0}-y\right|}{\left|x-x_{0}\right|^{n+1}}|a(y)| d y \\
& \leq\left[\frac{1}{\left(\rho\left(x_{0}\right)\right)^{n}}+\frac{r}{\left|x-x_{0}\right|^{n+1}}\right]\|a\|_{L^{1}\left(\mathbb{R}^{n}\right)},
\end{aligned}
$$

where, in the penultimate inequality, we use the fact that if $|x-y|<\rho\left(x_{0}\right) / 2 b_{2}$, then $\eta(|x-y| / \rho(x))=1$. This implies that

$$
\begin{aligned}
& \int_{Q\left(x_{0}, b_{2} \rho\left(x_{0}\right)\right) \backslash Q\left(x_{0}, 2 r\right)}\left|R_{j}^{l}(a)(x)\right| \omega(x) d x \\
& \leq\|a\|_{L^{1}\left(\mathbb{R}^{n}\right)} \int_{\mathrm{Q}\left(x_{0}, b_{2} \rho\left(x_{0}\right)\right) \backslash \mathrm{Q}\left(x_{0}, 2 r\right)} \frac{\omega(x)}{\left(\rho\left(x_{0}\right)\right)^{n}} d x \\
& \quad+\|a\|_{L^{1}\left(\mathbb{R}^{n}\right)} \int_{\mathrm{Q}\left(x_{0}, b_{2} \rho\left(x_{0}\right)\right) \backslash \mathrm{Q}\left(x_{0}, 2 r\right)} \frac{r \omega(x)}{\left|x-x_{0}\right|^{n+1}} d x \\
& \equiv I+I I .
\end{aligned}
$$

For $I$, since supp $a \subset Q_{0} \equiv Q\left(x_{0}, r\right) \subset Q\left(x_{0}, \rho\left(x_{0}\right)\right) \equiv Q$, by Lemmas 4 and 7 , we have

$$
\begin{aligned}
I & \lesssim\|a\|_{L^{1}\left(\mathbb{R}^{n}\right)} \frac{\omega\left(Q\left(x_{0}, b_{2} \rho\left(x_{0}\right)\right)\right)}{\left(\rho\left(x_{0}\right)\right)^{n}} \\
& \lesssim \frac{|Q|}{\omega(Q)} \frac{\omega\left(Q\left(x_{0}, b_{2} \rho\left(x_{0}\right)\right)\right)}{\left(\rho\left(x_{0}\right)\right)^{n}} \lesssim 1 .
\end{aligned}
$$

For $I I$, by Lemmas 4 and 7 , we get

$$
\begin{aligned}
I I & \leqslant\|a\|_{L^{1}\left(\mathbb{R}^{n}\right)} \int_{2 r \leq\left|x-x_{0}\right| \leq b_{2} \rho\left(x_{0}\right)} \frac{r \omega(x)}{\left|x-x_{0}\right|^{n+1}} d x \\
& \leq\|a\|_{L^{1}\left(\mathbb{R}^{n}\right)} \sum_{j=2}^{j_{0}} \int_{\left|x-x_{0}\right| 2^{j} r} \frac{r \omega(x)}{\left|x-x_{0}\right|^{n+1}} d x \\
& \leq \frac{\left|Q_{0}\right|}{\omega\left(Q_{0}\right)} \sum_{j=2}^{j_{0}} 2^{-k} \frac{\omega\left(2^{j} Q_{0}\right)}{\left|2^{k} Q_{0}\right|} \lesssim 1,
\end{aligned}
$$

where $j_{0}$ is an integer such that $2^{j_{0}-1} r<b_{2} \rho\left(x_{0}\right) \leq 2^{j_{0}} r$. Thus, for $r<L_{2} \rho\left(x_{0}\right)$ we obtain

$$
\left\|R_{j}^{l}(a)\right\|_{L_{\omega}^{1}\left(\mathbb{R}^{n}\right)} \lesssim 1
$$

Finally, we prove $\|f\|_{L_{\omega}^{1}\left(\mathbb{R}^{n}\right)}+\sum_{j=1}^{n}\left\|R_{j}(f)\right\|_{L_{\omega}^{1}\left(\mathbb{R}^{n}\right)} \sim$ $\|f\|_{L_{\omega}^{1}\left(\mathbb{R}^{n}\right)}+\sum_{j=1}^{n}\left\|R_{j}^{l}(f)\right\|_{L_{\omega}^{1}\left(\mathbb{R}^{n}\right)}$. Indeed, we only need to show that

$$
\left\|R_{j}^{l}(f)-R_{j}(f)\right\|_{L_{\omega}^{1}\left(\mathbb{R}^{n}\right)} \leqslant\|f\|_{L_{\omega}^{1}\left(\mathbb{R}^{n}\right)} .
$$

In fact, note that $|x-y| \leq \rho(x)$; then there exists a constant $\beta>0$ such that $\rho(y) / \beta \leq \rho(x) \leq \beta \rho(y)$ by Lemma 1 . Set $\omega \epsilon$ $A_{1}^{\rho, \theta}\left(\mathbb{R}^{n}\right)$ with $\theta>0$. Hence, by the properties of $A_{1}^{\rho, \theta}\left(\mathbb{R}^{n}\right)$, we get

$$
\begin{aligned}
& \left\|R_{j}^{l}(f)-R_{j}(f)\right\|_{L_{\omega}^{1}\left(\mathbb{R}^{n}\right)} \leq \iint_{\mathbb{R}^{n}} \mid R_{j}(x, y)-\widetilde{R}_{j}(x, y) \\
& \cdot \eta\left(\frac{|x-y|}{\rho(x)}\right)|| f(y) \mid d y \omega(x) d x \\
& \leq \int_{\mathbb{R}^{n}} \int_{|x-y|<\rho(y) / \beta}\left|R_{j}(x, y)-\widetilde{R}_{j}(x, y)\right| \\
& \cdot \omega(x) d x|f(y)| d y
\end{aligned}
$$




$$
\begin{aligned}
& +\int_{\mathbb{R}^{n}} \int_{\rho(y) / \beta \leq|x-y|<\beta \rho(y)}\left|\widetilde{R}_{j}(x, y)\right| \\
& \cdot \omega(x) d x|f(y)| d y \\
& +\int_{\mathbb{R}^{n}} \int_{\rho(y) / \beta \leq|x-y|}\left|R_{j}(x, y)\right| \omega(x) d x|f(y)| d y \\
& \lesssim\|f\|_{L_{\omega}^{1}\left(\mathbb{R}^{n}\right)}+\int_{\mathbb{R}^{n}} \frac{\omega(Q(y, \rho(y)))}{(\rho(y))^{n}}|f(y)| d y \\
& +\int_{\mathbb{R}^{n}} \sum_{k=1}^{\infty} 2^{-k N}\left|2^{k} \rho(y)\right|^{-n} \\
& \cdot \int_{|x-y| \leq 2^{k} \rho(y) / \beta} \omega(x) d x|f(y)| d y \\
& \leq\|f\|_{L_{\omega}^{1}\left(\mathbb{R}^{n}\right)}+\int_{\mathbb{R}^{n}} \sum_{k=1}^{\infty} 2^{-k\left(N-\left(k_{0}+1\right) \theta\right)} \\
& +\frac{\omega(Q(y, \rho(y)))}{(\rho(y))^{n}}|f(y)| d y \leq\|f\|_{L_{\omega}^{1}\left(\mathbb{R}^{n}\right)},
\end{aligned}
$$

if taking $N>\left(k_{0}+1\right) \theta+1$.

Therefore, the proof is complete.

\section{Competing Interests}

The author declares that there are no competing interests regarding the publication of this paper.

\section{Acknowledgments}

The research is supported by National Natural Science Foundation of China under Grant no. 11271024 and no. 11426038, and it is also supported by Scientific Research Promotion Plan of 2016 of Beijing International Studies University under Grant no. 211017.

\section{References}

[1] J. Dziubański, G. Garrigós, T. Martínez, J. L. Torrea, and J. Zienkiewicz, "BMO spaces related to Schrödinger operators with potentials satisfying a reverse Höder inequality," Mathematische Zeitschrift, vol. 249, no. 2, pp. 329-356, 2005.

[2] Z. Shen, " $L^{p}$ estimates for Schrödinger operators with certain potentials," Annales de l'Institut Fourier, vol. 45, no. 2, pp. 513546, 1995.

[3] B. Bongioanni, E. Harboure, and O. Salinas, "Classes of weights related to Schrödinger operators," Journal of Mathematical Analysis and Applications, vol. 373, no. 2, pp. 563-579, 2011.

[4] B. Bongioanni, E. Harboure, and O. Salinas, "Commutators of Riesz transforms related to Schrödinger operators," The Journal of Fourier Analysis and Applications, vol. 17, no. 1, pp. 115-134, 2011.

[5] J. Dziubański and J. Zienkiewicz, "Hardy space H1 associated to Schrödinger operator with potential satisfying reverse Hölder inequality," Revista Matemática Iberoamericana, vol. 15, no. 2, pp. 279-296, 1999.
[6] J. Dziubański and J. Zienkiewicz, " $H^{p}$ spaces for Schrödinger operators, Fourier Analysis and Related Topics," Banach Center Publications, vol. 56, pp. 45-53, 2002.

[7] J. Dziubański and J. Zienkiewicz, " $H^{p}$ spaces associated with Schrödinger operators with potentials from reverse Hölder classes," Colloquium Mathematicum, vol. 98, no. 1, pp. 5-38, 2003.

[8] H. Liu, L. Tang, and H. Zhu, "Weighted Hardy spaces and BMO spaces associated with Schrödinger operators," Mathematische Nachrichten, vol. 285, no. 17-18, pp. 2173-2207, 2012.

[9] L. Tang, "Weighted norm inequalities for Schrödinger type operators," Forum Mathematicum, vol. 27, no. 4, pp. 2491-2532, 2015.

[10] L. Tang, "Extrapolation from $A_{\infty}^{\rho, \infty}$, vector-valued inequalities and applications in the Schrödinger settings," Arkiv för Matematik, vol. 52, no. 1, pp. 175-202, 2014.

[11] D. Yang and Y. Zhou, "Boundedness of sublinear operators in Hardy spaces on RD-spaces via atoms," Journal of Mathematical Analysis and Applications, vol. 339, no. 1, pp. 622-635, 2008.

[12] D. Yang and Y. Zhou, "Localized Hardy spaces $\mathrm{H}^{1}$ related to admissible functions on $\mathrm{RD}$-spaces and applications to Schrödinger operators," Transactions of the American Mathematical Society, vol. 363, no. 3, pp. 1197-1239, 2011.

[13] J. Zhong, Harmonic analysis for some Schrödinger type operators [Ph.D. thesis], Princeton University, 1993.

[14] H. Zhu and H. Liu, "Weighted estimates for bilinear operators," Journal of Function Spaces, vol. 2014, Article ID 797956, 10 pages, 2014.

[15] J. Duoandikoetxea, Fourier Analysis, vol. 29 of Graduate Studies in Mathematics, American Mathematical Society, Providence, RI, USA, 2000.

[16] J. García-Cuerva and J. Rubio de Francia, Weighted norm inequalities and related topics, vol. 116 of North-Holland Mathematics Studies, North-Holland, Amsterdam, The Netherlands, 1985.

[17] J. García-Cuerva, "Weighted $H^{p}$ spaces," Dissertationes Mathematicae, vol. 162, pp. 1-63, 1979.

[18] E. M. Stein, Harmonic Analysis: Real-variable Methods, Orthogonality, and Oscillatory Integrals, vol. 43 of Princeton Mathematical Series, Princeton University Press, Princeton, NJ, USA, 1993.

[19] J.-O. Stromberg and A. Torchinsky, Weighted Hardy Spaces, vol. 1381 of Lecture Notes in Mathematics, Springer, Berlin, Germany, 1989.

[20] L. Tang, "Weighted norm inequalities for pseudo-differential operators with smooth symbols and their commutators," Journal of Functional Analysis, vol. 262, no. 4, pp. 1603-1629, 2012.

[21] H. Zhu and L. Tang, "Weighted local Hardy spaces associated to Schrödinger operators," http://arxiv.org/abs/1403.7641.

[22] D. Yang, D. Yang, and Y. Zhou, "Endpoint properties of localized Riesz transforms and fractional integrals associated to Schrödinger operators," Potential Analysis, vol. 30, no. 3, pp. 271-300, 2009.

[23] G. Mauceri, M. A. Picardello, and F. Ricci, "A Hardy space associated with twisted convolution," Advances in Mathematics, vol. 39, no. 3, pp. 270-288, 1981.

[24] B. Bongioanni, E. Harboure, and O. Salinas, "Riesz transforms related to Schrödinger operators acting on BMO type spaces," Journal of Mathematical Analysis and Applications, vol. 357, no. 1, pp. 115-131, 2009.

[25] F. W. Gehring, "The $L^{p}$-integrability of the partial derivatives of a quasi-conformal mapping," Acta Mathematica, vol. 130, pp. 265-277, 1973. 


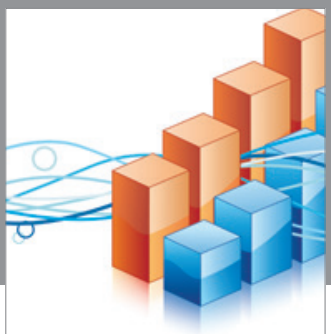

Advances in

Operations Research

vatem alat4

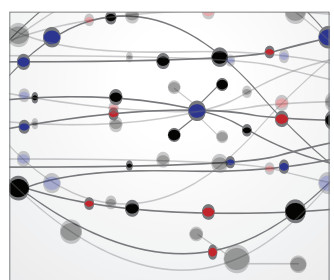

\section{The Scientific} World Journal
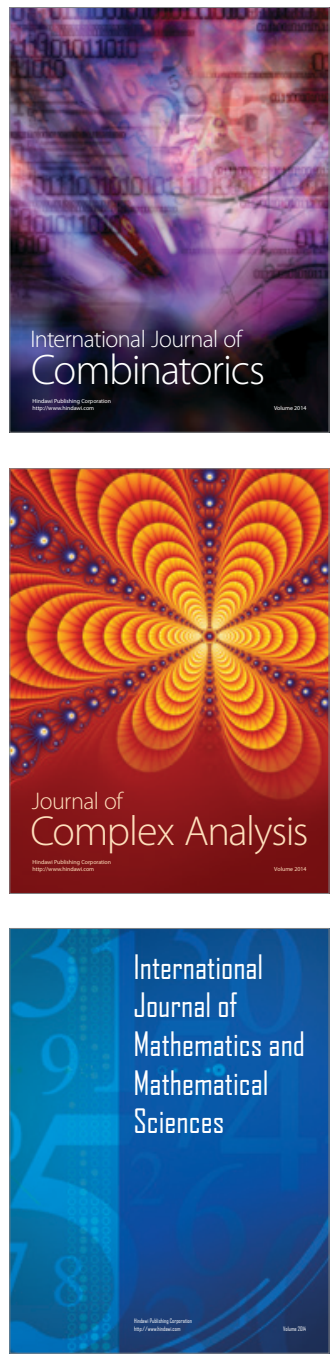
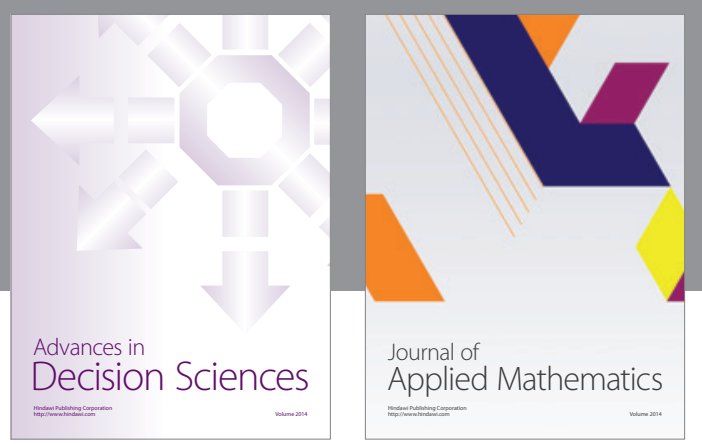

Algebra

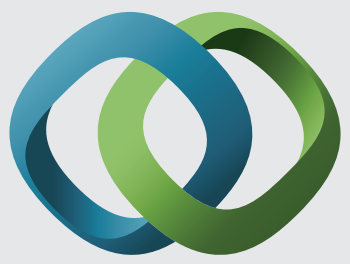

\section{Hindawi}

Submit your manuscripts at

http://www.hindawi.com
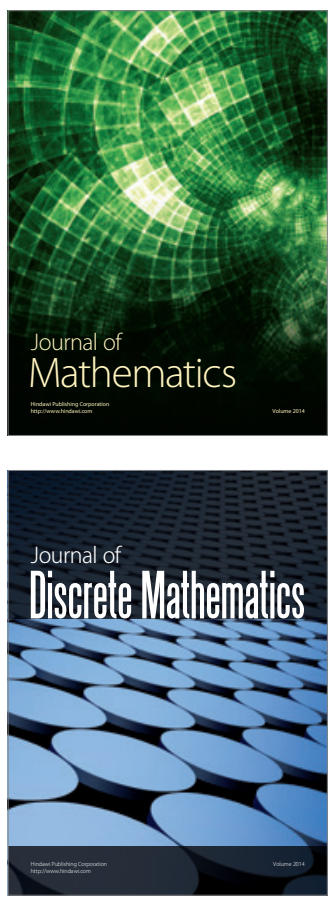

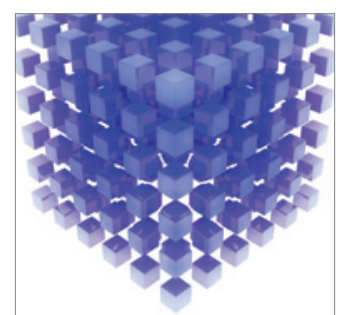

Mathematical Problems in Engineering
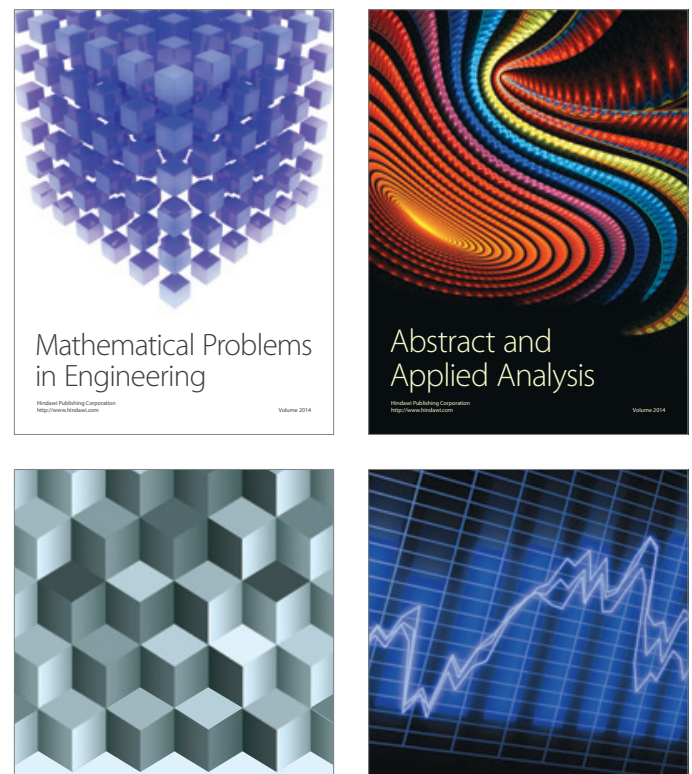

Journal of

Function Spaces

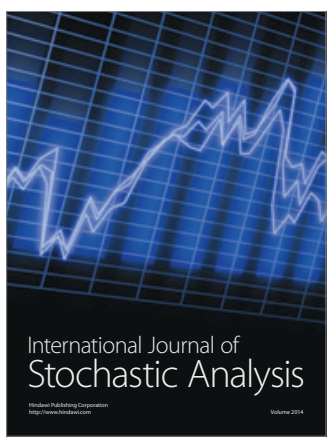

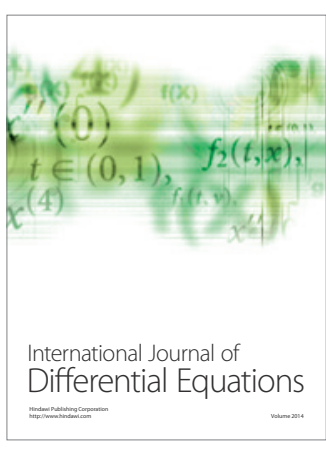
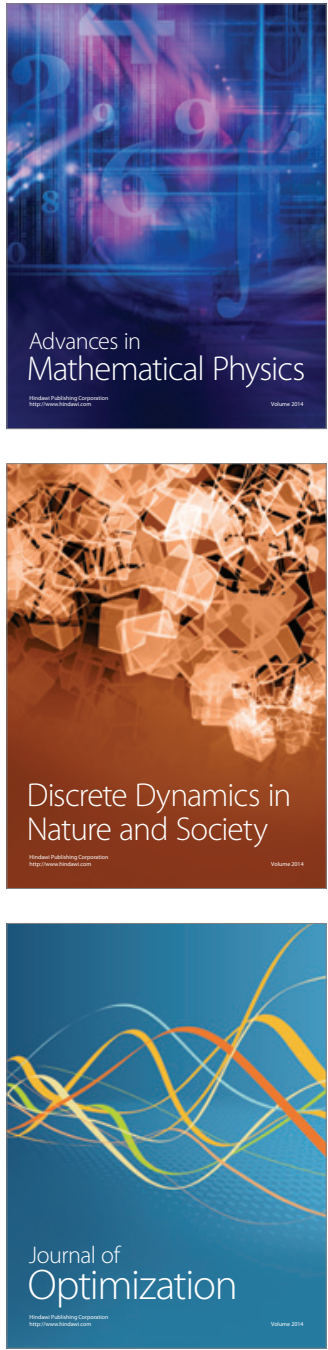